\title{
A Feasibility Study of the WHO Digital Mental Health Intervention Step-by-Step to Address Depression Among Chinese Young Adults
}

\begin{abstract}
Hao Fong Sit ${ }^{1}$, leng Wai Hong ${ }^{2}$, Sebastian Burchert ${ }^{3}$, Elvo Kuai Long Sou ${ }^{4}$, Mek Wong ${ }^{4}$, Wen Chen ${ }^{5}$, Agnes lok Fong Lam ${ }^{6}$ and Brian J. Hall ${ }^{7,8 *}$

${ }^{1}$ Department of Psychology, The University of Hong Kong, Pokfulam, Hong Kong SAR, China, ${ }^{2}$ Moon Chun Memorial College, University of Macau, Macau, Macau SAR, China, ${ }^{3}$ Department of Education and Psychology, Division of Clinical Psychological Intervention, Freie Universität Berlin, Berlin, Germany, ${ }^{4}$ Student Affairs Office, University of Macau, Macau, Macau SAR, China, ${ }^{5}$ Department of Medical Statistics, School of Public Health, Sun Yat-sen University, Guangzhou, China, ${ }^{6}$ Centre for Macau Studies, University of Macau, Macau, Macau SAR, China, ${ }^{7}$ Center for Global Health Equity, NYU Shanghai, Shanghai, China, ${ }^{8}$ Department of Health, Behavior, and Society, Johns Hopkins Bloomberg School of Public Health, Baltimore, MD, United States
\end{abstract}

OPEN ACCESS

Edited by:

Michael Patrick Schaub,

University of Zurich, Switzerland

Reviewed by:

Kim Mathiasen,

University of Southern

Denmark, Denmark

Karin Waldherr,

Ferdinand Porsche FernFH - Distance

Learning University of Applied

Sciences, Austria

*Correspondence:

Brian J. Hall

brianhall@nyu.edu

Specialty section:

This article was submitted to

Digital Mental Health,

a section of the journal

Frontiers in Psychiatry

Received: 10 November 2021 Accepted: 13 December 2021

Published: 07 January 2022

Citation:

Sit HF, Hong IW, Burchert S, Sou EKL,

Wong M, Chen W, Lam AlF and Hall BJ (2022) A Feasibility Study of

the WHO Digital Mental Health Intervention Step-by-Step to Address

Depression Among Chinese Young

Adults. Front. Psychiatry 12:812667.

doi: 10.3389/fpsyt.2021.812667
Background: Chinese young adults experience barriers to mental health treatment, including the lack of treatment providers and stigma around treatment seeking. Evidence-based digital mental health interventions are promising and scalable alternatives to face-to-face treatment for this population, but lack rigorous evidence to support scale-up in China.

Aim: The study was a feasibility study for a large-scale RCT of Step-by-Step, a behavioral activation-based, mental health intervention to address depression and anxiety symptoms in Chinese young adults. It sought to assess feasibility of recruitment and of delivery of Step-by-Step in a University setting, to assess acceptability of the intervention, and to examine potential effectiveness.

Method: An uncontrolled, feasibility trial was conducted to assess the feasibility and acceptability of Chinese Step-by-Step for Chinese University students with elevated depressive symptoms (PHQ-9 scores at or above 10) in Macao, China. Data was collected at two different time points (i.e., baseline and 8-weeks after baseline), administered via questionnaires embedded in an interventional mobile application. Participation rate and dropout rate were measured. Depressive and anxiety symptom severity, well-being, and self-defined stress were assessed. Satisfaction with the program was assessed using qualitative interviews.

Results: A total of 173 students were screened, 22.0\% $(n=38)$ were eligible, and $63.2 \%$ of them $(n=24)$ started the intervention. The dropout rate by post-test was $45.8 \%$. Results from completers showed that Step-by-Step was potentially effective in reducing depressive and anxiety symptom severity, and self-defined stress. Students were generally satisfied with the program, but also offered suggestions for continued improvement. Qualitative feedback was reported within the RE-AIM framework, covering recruitment, effectiveness, adoption, implementation, and maintenance. Amendments 
to the program were made according to the feedback (e.g., adding notification for new session, modify the time zone).

Conclusion: A minimally guided Step-by-Step protocol and the study procedure were successfully pilot tested for use for Chinese University students. The intervention was acceptable and no adverse events were reported. The results support the potential effectiveness and feasibility of a large-scale evaluation of the program.

Keywords: depression, digital health, e-mental health, minimally guided intervention, feasibility, Chinese young adults

\section{INTRODUCTION}

Evidence-based digital mental health interventions have gained empirical support and their efficacy is widely supported (1-4). This novel delivery model can reduce the treatment gap (5) and stigma-related barriers to seeking mental health treatment $(6,7)$, but few rigorous studies on digital mental health interventions were conducted within low- and middle-income countries (LMIC) (8), and among Chinese people in particular. Evidence from a meta-analysis of 22 randomized controlled trials including 4,104 participants showed that digital mental health interventions were effective in alleviating symptoms of depression, anxiety, and post-traumatic stress disorder, with a moderate to large effect size (8). In the past decade, there was an increase in digital mental health interventions for Chinese, but few were developed or adapted for depression, and even fewer addressed depression through smartphone applications, according to a recent systematic review on digital mental health in mainland China (9).

Emerging adulthood is a crucial developmental stage when individuals are undergoing complex and dynamic changes (10), with increased vulnerability to poor mental health because of the developmental, academic, occupational, and social challenges they encounter $(10,11)$. University students, a large community of young adults, are at risk of a variety of mental health issues, and depression and anxiety are common in this population (1115). In China, roughly $30 \%$ of college students have clinically significant depression (14). Such high prevalence was also witnessed during the COVID-19 pandemic (16), and a shift of delivery model from conventional approaches (e.g., face-to-face) to digital treatment (e.g., through digital devices) has increased the public's awareness. However, evidence for the use of digital mental health interventions for depression in China is still lacking, and the scalability and implementation of these services is uncertain.

To improve access to interventions globally, the World Health Organization (WHO) developed technology-assisted interventions that have the potential to scale (17). In collaboration with the Ministry of Public Health in Lebanon, University of Zurich, Switzerland, and Freie Universität Berlin,

Abbreviations: CONSORT, Consolidated Standards of Reporting Trials; GAD-7, The General Anxiety Disorder-7; LMIC, low- and middle-income countries; PHQ9, The Patient Health Questionnaire-9; PSYCHOLOPS, Psychological Outcome Profiles; SbS, Step-by-Step; WHO, World Health Organization; WHO-5, The World Health Organization-Five Well-Being Index.
Germany, the WHO developed the web version of Step-by-Step (SbS) (17), which was later developed into a mobile app and culturally adapted for a diversity of populations (18-23). In order to evaluate the effectiveness and implementation of SbS in a Chinese tertiary educational setting, SbS was culturally adapted (23), followed by the current feasibility study that preliminarily tested the adapted intervention, and informed the study process of a future randomized controlled trial (RCT), which is now planned (24), guided by the developmentevaluation-implementation process (25) and implementation evaluation framework $(26,27)$.

The objectives of the current study were to assess the feasibility of delivering Step-by-Step in a University setting for Chinese young adults utilizing a minimal peer-support guidance model and obtain preliminary evidence of the effectiveness of the intervention to reduce depressive and anxiety symptom severity.

\section{METHODS}

\section{Trial Design}

An uncontrolled, single-arm pilot trial was conducted to understand the feasibility and acceptability of Chinese Step-byStep for University students with elevated depressive symptoms in Macao (SAR), China. Data were collected at two different time points (i.e., baseline and 8-weeks after baseline), administered via questionnaires embedded in the interventional mobile application. Although the current feasibility study adopted an uncontrolled design, it followed the CONSORT statement (28).

\section{Participants \\ Eligible Criteria}

Participants were included if they had a score of Patient Health Questionnaire-9 (PHQ-9) of 10 or higher, were students, at least 18 years old, Chinese nationality, Cantonese or Mandarinspeaking, had a permit to stay in Macao, and had a digital device to access the intervention (e.g., smartphone). Participants were excluded if they reported high levels of suicidal ideation or a plan to self-harm in the past month.

\section{Setting and Recruitment}

The University of Macau is the largest public University in Macao. It hosts roughly 10,000 local and mainland Chinese students. It has a residential college system where students reside and participate in co-curricular and general education program. Moon Chun Memorial College, one of the 10 residential 
colleges, houses approximately 500 students, and served as a context for participant recruitment and data collection for this feasibility study. Multiple strategies were adopted for recruitment within the residential college, including posters, mass email invitations, referrals, in-class recruitment, posts in social networking application, and a booth in residential college events.

All eligible participants in the feasibility study were allocated to the 5-session Step-by-Step intervention to test the study procedure, and acceptability of adapted content, and obtain evidence of potential intervention effectiveness. SbS was delivered via a smartphone application using iOS and Android systems or a website that can be accessed on digital devices (e.g., smartphone, laptop).

\section{Intervention}

$\mathrm{SbS}$ is a 5 -session illustrated narrative program designed to be transdiagnostic with a primary focus on depression, which includes components of psychoeducation, relaxation techniques, identifying personal strengths, positive self-talk, enhancing social support, and relapse prevention, with behavioral activation as the core therapeutic component and based on WHO guidelines and recommendations $(17,29)$. It is delivered in an 8 -week timeframe through digital devices. Before entering the five therapeutic sessions, each user went through an onboarding session in the app, which explained the study, obtained consent, and confirmed eligibility. Consent items were presented one by one. The consent progress only proceeded when the participant chose "agree," or the process of consent and the program ended if "disagree" were chosen. Eligible participants entered an introduction session (session 0) consisting of an introduction to SbS sessions and a baseline assessment. Each therapeutic session consisted of 2-3 parts with illustrated narratives and exercises and lasted around 20-30 min. Participants were recommended to finish one session per week before a new session was unlocked. Three main types of exercises were provided in the application, including behavioral activation (e.g., activity scheduling), self-care (e.g., gratitude exercise), and relaxation (e.g., breathing exercise). The mood tracking feature allowed users to record their mood every day. Each participant is assigned an e-helper, a trained non-specialist who provides minimal support by phone and text to participants. E-helpers were responsible for check-in with the participants, motivate them to complete one session per week, and solve technique problems concerning using the app on a weekly basis. Each e-helper handled no more than four active users at the same time. After the 8 week of intervention period, regardless whether the five sessions were completed or not, participants were invited to interview, and they could go on learning the remaining sessions if they had not finished within 8 weeks and using the exercises in the app.

\section{E-Helper Training}

As part of the program, e-helpers provided minimal weekly guidance. In this pilot study, we trained peers to offer this guidance, as this was deemed to be most likely to support scalability and maintenance of the program. E-helpers were unpaid undergraduate peer counselors from our partner residential college, undergraduate research assistants, and a masters-level counseling and psychotherapy postgraduate interns from the Students Affairs Office. E-helper training was provided by the psychological counselors from the University and the study coordinator from the research team. The 24-h training covered mental health first-aid (12 h), an overview of Step-byStep, clinical training on how to communicate during weekly contacts $(9 \mathrm{~h})$, and technical training on the mobile application $(3 \mathrm{~h})$, was held during the weekend or weekday evenings to fit the e-helpers' schedule. The sub-set of student peer counselor ehelpers $(n=13)$ who were recruited for this study were evaluated using performance-based role-play exercises before they were deemed appropriate to provide guidance to other students. All ehelpers received weekly 1 -h group supervision during the study intervention period.

\section{Measures Overview}

Participant sociodemographic information including age, sex, educational level, marital status, place of birth, identification for staying in Macao and previous psychological or mental health service use (Yes/No), were collected. Feasibility and acceptability of the intervention were evaluated by rate of participation and dropout, and the process of intervention implementation that with a focus on recruitment strategy, data collection tool, and intervention adoption (30).

\section{Primary Outcome Measure}

The Chinese version of the self-reported Patient Health Questionnaire-9 (PHQ-9) (31), was used to measure depression symptom severity with a four-point scale, ranging from 0 (not at all) to 3 (nearly every day) and higher scores indicating higher depressive symptoms (0-27). A cut-off score of 10 was used to detect the clinical presence of depression in our sample $(32,33)$. The Chinese PHQ- 9 was validated and showed excellent internal consistency in the Chinese population $(\alpha>0.80)(32-34)$.

\section{Secondary Outcome Measures}

The Chinese version of the General Anxiety Disorder-7 (GAD7) (35-37) and World Health Organization-Five Well-Being Index $(\mathrm{WHO}-5)(38,39)$ were used to evaluate anxiety and well-being and demonstrated good reliability $(\alpha s>0.80)$ and validity in Chinese populations. The Psychological Outcome Profiles (PSYCHOLOPS) (40) was used to evaluate clientdefined stress and was translated from the original English version by a Chinese-English bilingual research assistant, and a backward translation was done by another Chinese-English research assistant to ensure the items are cultural and conceptual equivalent in the study.

\section{Implementation Evaluation}

A qualitative interview guide was adapted from another digital mental health project (21) and further modified based on the RE-AIM framework $(26,27)$. The RE-AIM framework has 5 dimensions, including recruitment ("How do I reach the targeted population with the intervention?"), efficacy or effectiveness ("How do I know the intervention is efficacious 
or effective?"), adoption ("How do I develop organizational support to deliver the intervention?"), implementation ("How do I ensure the intervention is delivered properly?"), and maintenance ("How do I incorporate the intervention for its long term delivery?") $(26,27)$. The interview guide was used to evaluate the process of the study covering the following aspects: recruitment, the effect of the intervention, implementation, adoption, and maintenance (Appendix 1 in Supplementary Material).

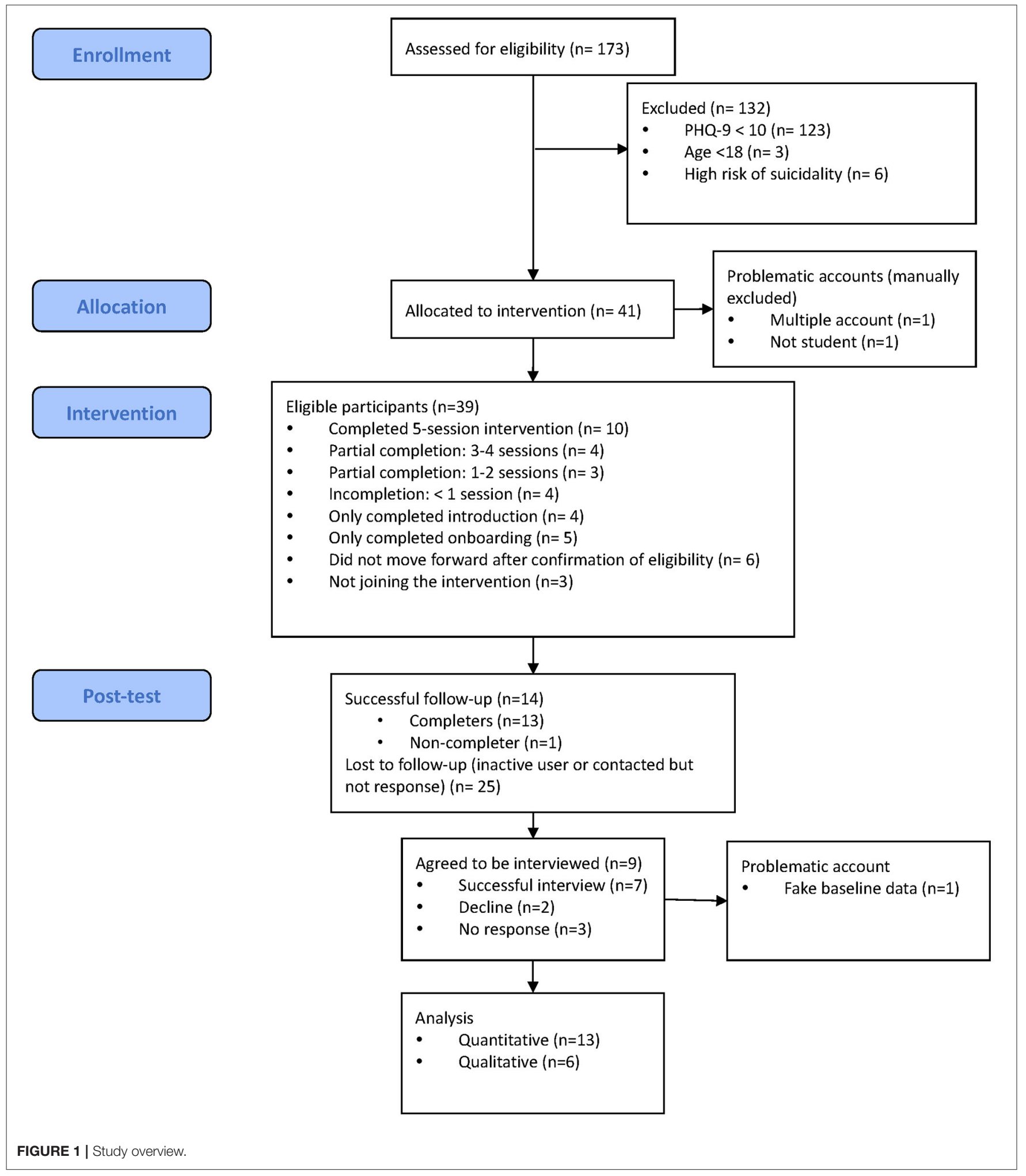


Since Step-by-Step was a minimal-guided, digital, self-help intervention, participants were considered as the major agent regarding intervention adoption.

\section{Dropout and Adherence}

A record of dropout, reason of dropout, and a number of completed sessions were recorded throughout the study. Reasons of dropout were obtained from the weekly contact with the participant by e-helpers.

\section{Analysis}

\section{Statistical Analysis}

Descriptive statistics (mean and standard deviation for continuous variables and frequency and proportions for categorical variables) described participants' demographic, clinical characteristics, and treatment adherence. Treatment outcomes were graphically summarized across time points. Paired sample $t$-tests were performed to obtain within-group change in study outcomes from baseline to post-test assessment. For the purpose of our analyses and comparison with previous studies $(20,41)$, we considered completers to be anyone who at least partially completed session 0 and 3 additional sessions, starters to be anyone who at least partially completed session 0 and completed 1-2 additional sessions, and non-starters to be anyone who completed none of the interventional sessions (i.e., session 1-5). Both starters and non-starters who completed session 0 were considered as dropouts. We used Stata 16 for Windows (42) for the analysis of the data.

\section{Qualitative Analysis}

Content analysis was applied to identify and describe the five dimensions of the RE-AIM framework $(26,27)$. This framework is applied to understand key dimensions for the implementation of evidence-based interventions. We adopted this framework to allow us to gather additional information on the longterm maintenance and scalability of the program following a randomized controlled trial. All interviews were transcribed verbatim and translated from Chinese to English, and two assistants coded each transcript independently. The two coders reached a consensus on the understanding of each code by discussion and invited a third coder to decide the code for the context when consensus could not be reached.

\section{Monitoring Harms}

Harm is defined as a sustained deterioration directly caused by the intervention (43). The PHQ-9 was used to monitor participants' depressive symptoms weekly, which informed ehelpers about the support that needed to be offered. In addition, if any adverse event (e.g., self-harm, symptom deterioration, distress) occurred, the e-helper would report to the clinical supervisor of the e-helper management team. A referral would be made for participants who reported any life-threatening event (i.e., self-harm, suicidal attempt) to the University counseling center where a professional team of social workers, psychological counselors, and psychotherapists could provide support, including referrals to additional services.

\section{Ethics and Consent}

The study obtained ethics approval at the University of Macau (MYRG2018-00241-FSS), and all participants provided consent to participate in the study.

\section{RESULTS}

\section{Sample Descriptions}

Details of the study overview are shown in Figure 1. A total of 173 participants were assessed for eligibility between October 2020 and November 2020. One hundred and thirty-two participants (76.3\%) were excluded because of low levels of depressive symptoms ( $n=123,71.1 \%)$, aged below 18 ( $n=3,1.7 \%)$, and high risk of suicidality $(n=6,3.5 \%)$. Among the excluded participants, most of them were female $(n=68,54.5 \%)$, and local students $(n=76,57.6 \%)$.

Forty-one users were eligible. Among them, three accounts were removed (one account was not functional, one was not a student, and one was removed during analysis as it was discovered they had reported high symptoms to enter the trial). Figure 1 showed the participant flow. Figure 2 showed the number of participants who completed each session. A total of 38 eligible participants were included, 14 did not start any session of SbS ( 5 only answered the baseline survey [on boarding session], 6 did not try any part of a session after confirmation of eligibility, and 3 self-excluded). Among the 24 participants who started SbS, 4 only completed the introduction session [session 0], 4 tried some parts of session 1, 3 completed 1-2 sessions, 4 completed 3-4 sessions (including one participant who completed 2 of the 3 parts in session 3), and 9 completed the 5 -session intervention. Based on our definition of non-starter (see section Statistical Analysis), 15 non-starters were identified, including 5 only completing the onboarding session, 6 trying no part of a session after confirmation of eligibility, and 4 only completing the introduction session (session 0). Among those who started any session of SbS (including session 0), 11 participants who completed fewer than 3 sessions (45.8\%) were considered dropouts and 13 participants (54.2\%) were considered completers (completed at least three therapeutic sessions). Reasons for drop-out included insufficient time due a

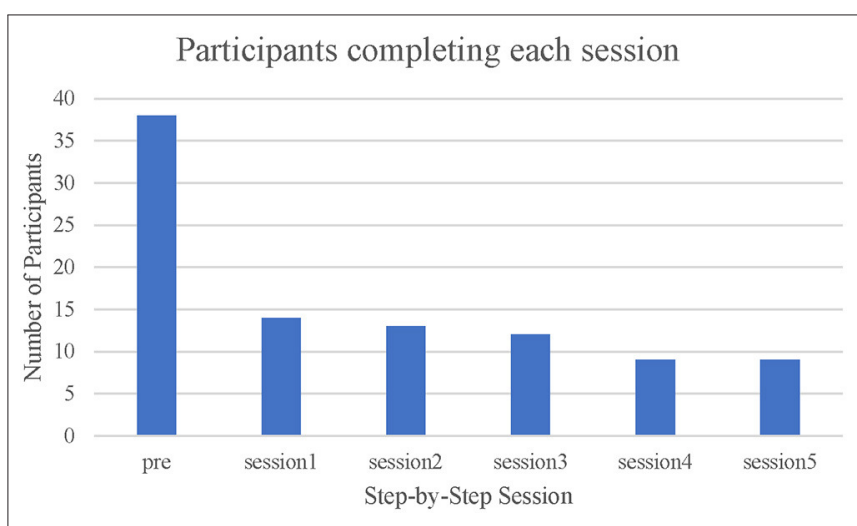

FIGURE 2 | Number of participants completing each session. 
TABLE 1 | Participant characteristics at baseline $(N=38)$.

\begin{tabular}{|c|c|c|c|c|}
\hline & $\begin{array}{c}\text { Completers }(n=13) \\
n(\%)\end{array}$ & $\begin{array}{c}\text { Starters }(n=7) \\
n(\%)\end{array}$ & $\begin{array}{c}\text { Non-starters }(n=15) \\
n(\%)\end{array}$ & $\begin{array}{c}\text { Self-excluded }(n=3) \\
n(\%)\end{array}$ \\
\hline Age $(18-25, M, S D)$ & $19.00(0.91)$ & $20.14(2.19)$ & $18.46(0.66)$ & $18(0)$ \\
\hline Male & $3(7.9 \%)$ & $3(7.9 \%)$ & $2(5.3 \%)$ & $3(7.9 \%)$ \\
\hline Female & $10(26.3 \%)$ & $4(10.5 \%)$ & $12(31.6 \%)$ & - \\
\hline Year 2 & $3(7.9 \%)$ & $2(5.3 \%)$ & $1(2.6 \%)$ & - \\
\hline Year 3 & $2(5.3 \%)$ & $1(2.6 \%)$ & $1(2.6 \%)$ & - \\
\hline Year 4 & $1(2.6 \%)$ & $2(5.3 \%)$ & - & - \\
\hline \multicolumn{5}{|l|}{ Born in Macau } \\
\hline Yes & $6(15.8 \%)$ & $2(5.3 \%)$ & $5(13.2 \%)$ & $1(2.6 \%)$ \\
\hline No & $2(7.7 \%)$ & $4(10.5 \%)$ & $5(13.2 \%)$ & - \\
\hline \multicolumn{5}{|l|}{ From where to know about SbS } \\
\hline Residential fellow (RF) & $10(26.3 \%)$ & $7(18.4 \%)$ & $9(23.7 \%)$ & $1(2.6 \%)$ \\
\hline Student counsellor/social worker & $1(2.6 \%)$ & - & - & - \\
\hline Friend & - & - & $2(5.3 \%)$ & - \\
\hline Organization/NGO & $1(2.6 \%)$ & - & $1(2.6 \%)$ & - \\
\hline Others & $1(2.6 \%)$ & - & $1(2.6 \%)$ & - \\
\hline \multicolumn{5}{|c|}{ Mental health service in the last 3 months (baseline) } \\
\hline Yes & $1(2.6 \%)$ & $3(7.9 \%)$ & $1(2.6 \%)$ & - \\
\hline No & $12(31.6 \%)$ & $4(10.5 \%)$ & $11(28.9 \%)$ & $1(2.6 \%)$ \\
\hline \multicolumn{5}{|c|}{ Mental disorders diagnosis in the last three months (baseline) } \\
\hline No & $12(31.6 \%)$ & $6(15.4 \%)$ & $12(31.6 \%)$ & $1(2.6 \%)$ \\
\hline
\end{tabular}

busy academic timetable, joining the program only for e-helper support, and not being aware that they needed to complete the intervention in 8 weeks. One completer did not complete the post-test questionnaire. No self-harm, symptom deterioration, increased distress, or suicidal attempt was reported. Details of their demographic characteristics and study outcomes are shown in Tables 1, 2.

For completer sub-group analyses, the 8 of the 13 participants who answered the post-test assessment completed the intervention were categorized as full completers $(n=8)$, and 4 who did some sessions as partial completers $(n=4)$, and 1 person who did not complete any interventional session were considered as a control, for non-statistical comparison.

\section{Primary Outcomes}

Twelve participants who did the intervention and completed post-intervention assessment were included in the completer analyses. Mean, SD, correlation coefficient $(r)$, and effect size (Cohen's $d$ ) for PHQ-9, GAD-7, WHO-5, and PSYCHOLOPS are shown in Table 3.

A significant decrease of PHQ-9 scores was observed $\left[t_{(11)}=\right.$ 4.29, $p=0.001$, Cohen's $d=1.24$, two-tailed]. Mean and SD of PHQ-9 scores at baseline and post-assessment among control, partial completers and completers are displayed in Table 4. Completers demonstrated the greatest reduction, followed by partial completers and control (see also Figures 3, 4).

\section{Secondary Outcomes}

The complete case analysis showed a significant decrease of anxiety symptoms as measured with GAD-7 ( $p=0.024$, Cohen's $d=0.754$, two-tailed) and self-defined problems as measured with PSYCHOLOPS ( $p=0.005$. Cohen's $d=0.99$, two-tailed). A slight and non-statistically significant increase was observed for subjective well-being, $p=0.208$, Cohen's $d=0.386$, two-tailed. 
TABLE 2 | Study outcome variables at baseline $(N=38)$.

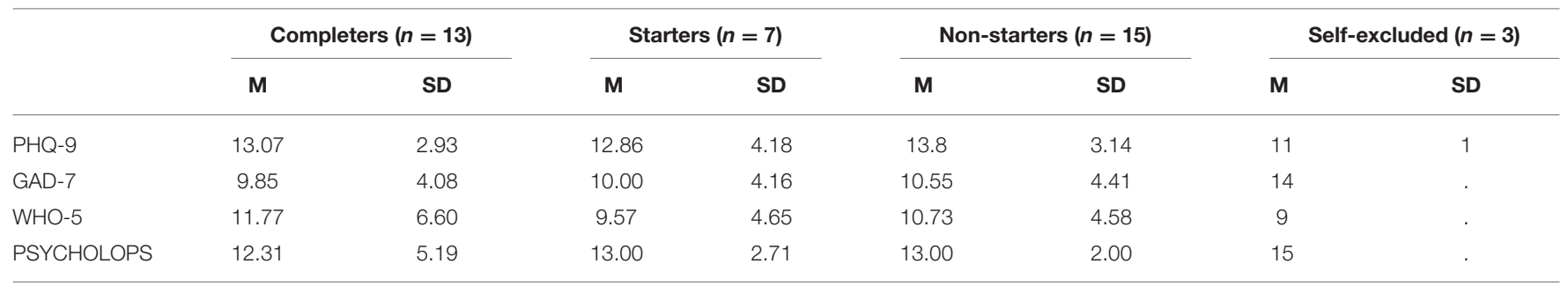

PHQ-9, The Patient Health Questionnaire-9; GAD-7, The General Anxiety Disorder-7; WHO-5, The World Health Organization- Five Well-Being Index; PSYCHLOPS, The Psychological Outcome Profiles. In Non-starters, 11 participants completed GAD-7 and WHO-5, and 7 completed PSYCHOLOPS. Among those who self-excluded, 1 participant completed GAD-7, WHO-5 and PSYCHOLOPS.

TABLE 3 | Baseline and post-test changes among completers.

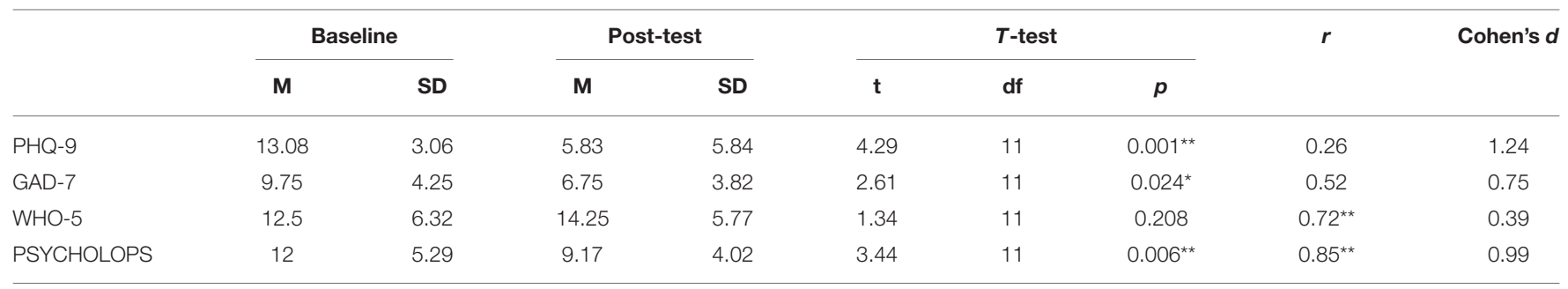

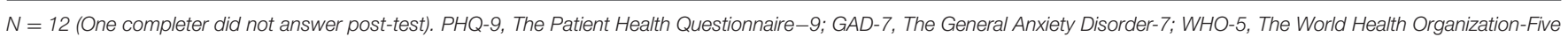
Well-Being Index; PSYCHLOPS, the Psychological Outcome Profiles. ${ }^{*} p<0.05,{ }^{\star \star} p<0.01$. Cohen's d: 0.2 (small), 0.5 (medium), 0.8 (large).

TABLE 4 | Means and SDs of clinical outcomes before and after intervention for control, partial completers and completers.

\begin{tabular}{|c|c|c|c|c|c|c|}
\hline & \multicolumn{2}{|c|}{ Control $(n=1)$} & \multicolumn{2}{|c|}{ Partial completers (3-4 sessions) $(n=4)$} & \multicolumn{2}{|c|}{ Full completers ( 5 sessions) $(n=8)$} \\
\hline PHQ-9 (M, SD) & 10 & 8 & $13.75(4.5)$ & $6.75(4.65)$ & $12.75(2.38)$ & $5.48(6.61)$ \\
\hline GAD-7 (M, SD) & 8 & 12 & $11.25(4.11)$ & $7.25(3.5)$ & $9.00(4.38)$ & $6.50(4.17)$ \\
\hline WHO-5 (M, SD) & 11 & 11 & $11.5(1.0)$ & $11.75(5.32)$ & $13.00(7.84)$ & $15.50(5.90)$ \\
\hline PSYCHLOPS (M, SD) & 9 & 12 & $12.75(4.5)$ & 10.75 (3.5) & $11.63(5.90)$ & $8.38(4.24)$ \\
\hline
\end{tabular}

PHQ-9, The Patient Health Questionnaire-9; GAD-7, The General Anxiety Disorder-7; WHO-5, The World Health Organization-Five Well-Being Index; PSYCHLOPS, the Psychological Outcome Profiles.

\section{Qualitative Process Evaluation}

Six participants were successfully contacted and willing to participate in the face-to-face qualitative interview.

\section{Reach}

Participants offered feedback and various suggestions to increase engagement and recruitment into subsequent trials. They agreed with the current strategy to recruit participants from within the residential college and the incentives we used. They suggested that utilizing social media channels (e.g., Facebook, Instagram, a WeChat official account), memes and other attractive marketing strategies, varying the content in simplified and traditional Chinese to attract the diverse student population, use of student groups, videos, and student email could increase recruitment. Additional feedback on the incentives was that bubble tea would be appropriate but stationary was not attractive. They also suggested that the use of a lucky draw that includes larger prizes can increase student engagement (e.g., tablet computers, video games consoles) in the recruitment.

\section{Adoption}

Adoption of intervention occurred at individual and organizational level. Participants suggested that the instructions were clear and the questionnaire length was appropriate, but the questions in the questionnaire are repeated (e.g., weekly assessment of depressive symptoms), so they may not have variation and the 2 -weeks interval.

\section{Impression}

There were differences between students' initial impressions about the app and what they later came to realize about its function. For example, several students expected that this app would be boring, similar to other app programs they experienced. They were surprised and engaged by the content. Others considered it to be worthwhile, innovative, but not dynamic, as it did not respond to user inputs. Another student thought 


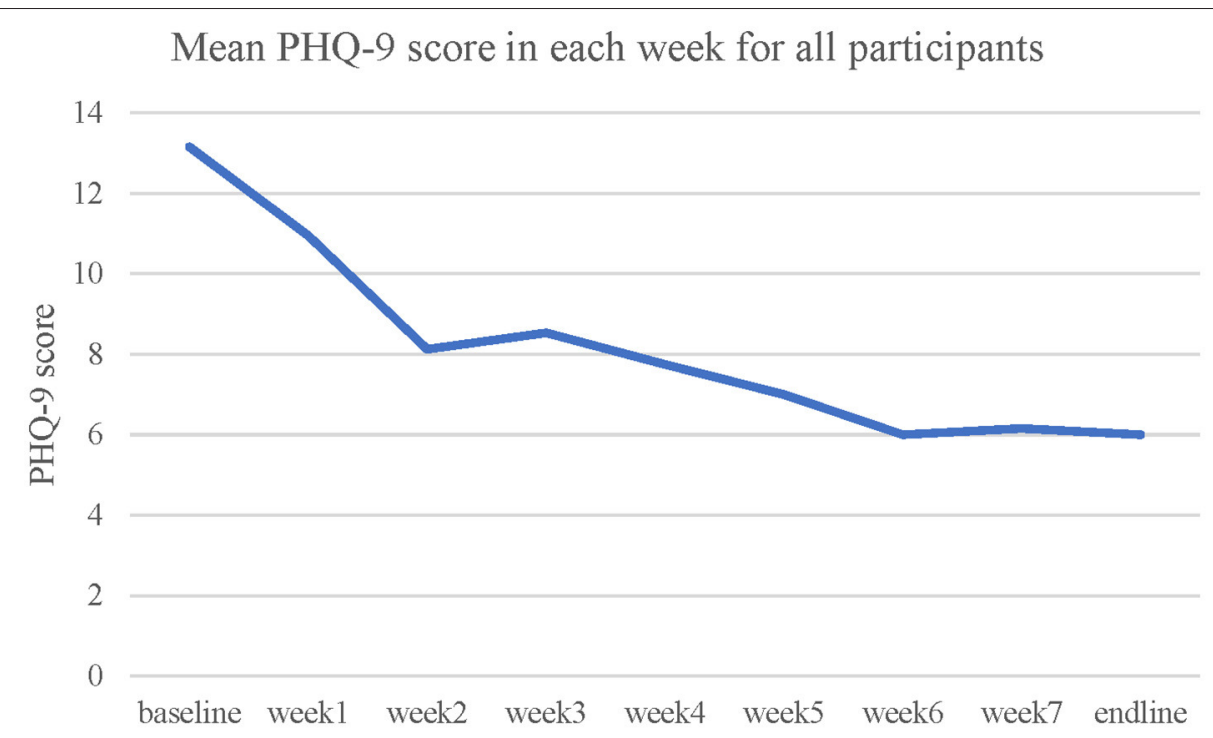

FIGURE 3 | Mean PHQ-9 score in each week for all participants across 8 weeks.

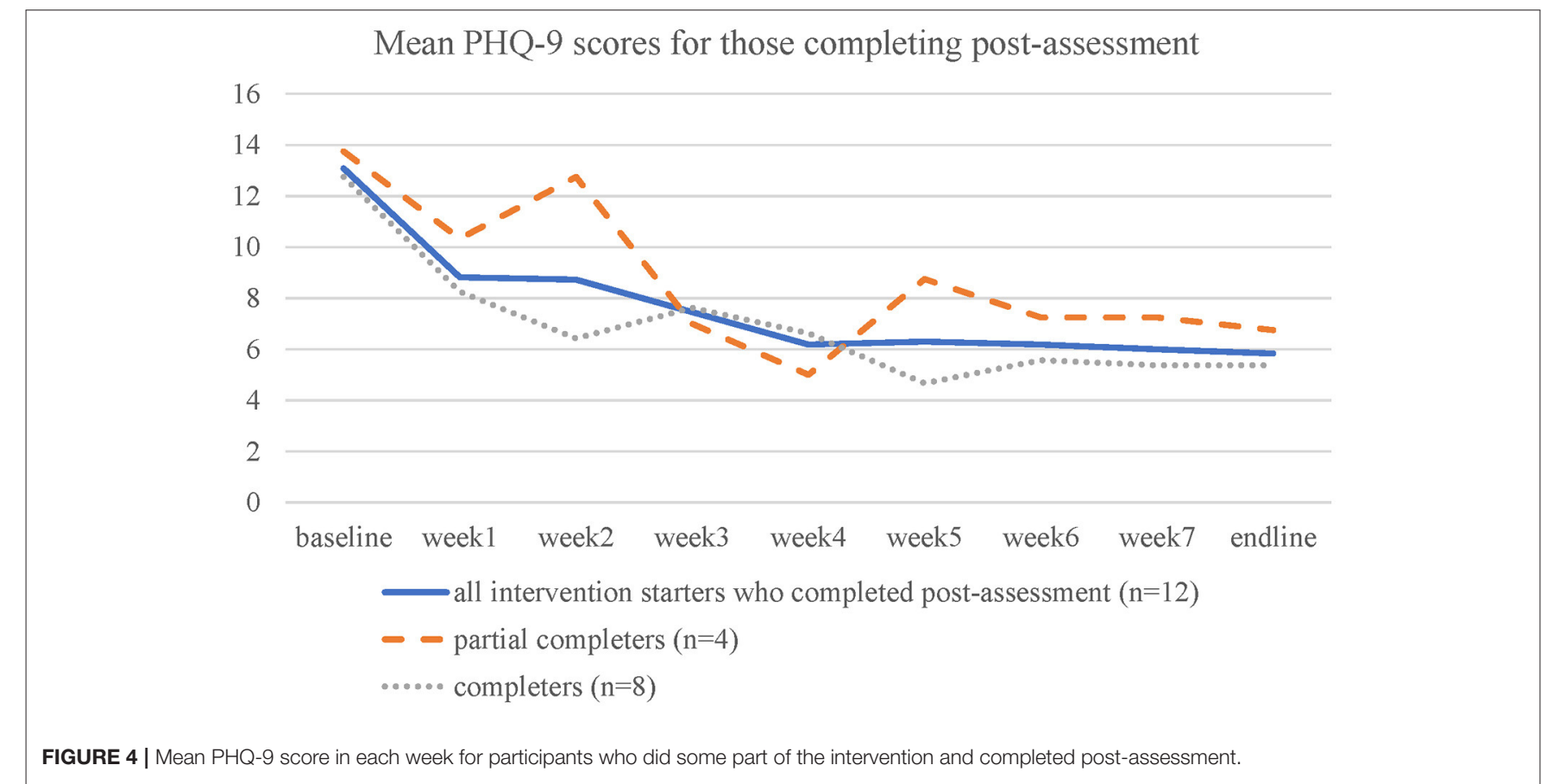

they would be speaking to a social worker, but then realized it was self-help.

\section{Time to Use}

Students varied with the time they used the app with several reporting late-night use, and others reporting use in the afternoon.

Students generally had positive feedback on the app. They considered it easy to use, with practical exercises, relatable stories, and engaging illustrated narrations, although a few students considered some scenarios to be less relevant or difficult to understand. Other concerns raised by the users about the app were that the audio exercise was too long that the user would get bored, app notifications were not frequent enough or occurred at an unwanted time.

Regarding organizational support, the SbS research team partnered with a residential college that was responsible for participants and e-helpers recruitment and the Student Counseling Section of the Student Affairs Office of the University that helped to train the e-helpers and provide regular supervision. 
The three parties worked closely during the feasibility study. Based on feedback from the interviewees, the recruitment activities were appropriate and sufficient and minimal guidance offered by e-helpers were helpful, which indicated organizational support was delivered well and led to the acceptance of recruitment strategies and guided support.

\section{Effectiveness}

Participants reported that they learned common stories about University students that remind them they were not alone, and further, they learned how to manage their emotions and schedule activities to help them relax and achieve their tasks. Activity scheduling, mood tracker, and relaxation exercise were most frequently used among the participants while a few participants found it difficult to master breathing exercises and were uncertain about what they could share in the gratitude exercise.

All participants received minimal support from e-helpers via a chatroom function in the app or phone calls, but not all participants availed themselves of this support because they did not encounter difficulties. For participants who were in need of support, they considered their e-helper helpful since they checked-in with them and listened to them. One participant reported that the e-helper prompted them to use the app and found this helpful.

Overall, participants were greatly satisfied with the program and the service they received, taking into account the function of the app, the improvement they perceived, and the knowledge or skills they acquired.

\section{Implementation}

Participants were satisfied with the SbS (range 7-8 out of 10, $\mathrm{M}=7.5$ ) regardless the number of completed sessions (range 3-5 sessions). Participants suggested a number of potential improvements to the program implementation. They suggested that the app could include videos as an alternative to illustrated narratives for those who preferred videos to text, and to add notifications when sessions were unlocked, accompanied by messages from their e-helper to keep themselves on track with the program, enhance notifications by making them visible similar to social networking applications, add additional timeslots for ehelpers to reply to user questions, add functions that facilitated interaction between the participant and characters and other study participants (e.g., group chat), add incentives within the app to encourage participants to engage in activities, and limit the amount of personal content requested by the app for the e-helper's monitoring.

All users universally reported a preference for female e-helpers for phone call support because of ease or willingness of disclosure of their problems to women.

\section{Maintenance}

Participants reflected several issues that should be addressed for the sustainability of the program. For instance, the app should avoid many open-ended questions in the questionnaire, provide sufficient information about the program (e.g., what participants are asked to do in the app) and sufficient notification to remind the participants to do the exercise or activities that they planned, offer timely responses by e-helpers, and provide additional assurances of confidentiality.

\section{DISCUSSION}

This feasibility study investigated the feasibility and acceptability of a culturally adapted, transdiagnostic mental health intervention to reduce depression among Chinese young adults. The study showed that the program was acceptable, feasible to implement, and promising given the reductions in symptoms observed among those who completed the intervention. Our study yielded a large effect size reduction in depressive symptoms and client-defined problem reduction and a medium effect size reduction of anxiety symptoms. Based on the qualitative feedback, the Chinese SbS showed a comparative satisfaction with another SbS program although a different evaluation was adopted (41). We also found a statistically non-significant trend of improvement in well-being. This study fills an important gap in the emerging evidence of the promise of app-based self-help within a Chinese population [for review, $(8,9)]$. Notwithstanding the feasibility design and recruitment strategy of the current study, the results suggest that the mental health of University students, especially freshmen, was poor. Using a PHQ-9 cutoff score of 10 to identify potential users in a residential college of the University, the inclusion rate was about $22.0 \%$ These results also support the expansion of more accessible resources and support to this population $(7,32)$.

Attrition in digital interventions is high and varies across studies $(44,45)$. A meta-analysis (46) estimated that the average dropout rate of digital intervention for depression was nearly $50 \%$. Due to the comparable participation rate with previous literature, we suggested that the Chinese SbS was feasible. Compared with Heim et al. (20) and Harper Shehadeh et al. (41), the non-starting rate (did not start any session) was $36.8 \%$ in this current study, which was lower than in other SbS studies (42.6 and $69.2 \%$ ). Overall dropout rate (completed $<4$ therapeutic sessions) among those who started the intervention $(n=24)$ was $45.8 \%$ at post-assessment in this study, which was similar to other SbS studies at approximately 50\%. People who did not start the program or dropped out from the study showed the greatest depressive and anxiety as well client-defined stress, compared with completers and starters at baseline (see Table 2). Their discontinuation may be due to the higher symptoms. The full program completion rate (completed all sessions) in this study was $37.5 \%(n=9)$ among intervention starters, which was similar to Harper Shehadeh et al. (41) and Heim et al. (20), 43.2 and $32.8 \%$, respectively. Completion rates (proportion of eligible participants who completed at least three therapeutic sessions in this study were comparable to other SbS studies, with $54.2 \%$ in the current study and $62.2 \%$ in (41) and $35.9 \%$ in Heim et al. (20). The preliminary results showed that full completers showed the greatest improvement, and minimal difference in improvement between full completers (completed all sessions) and partial completers (completed 3-4 sessions) was observed (see Table 4), suggesting the treatment gains between the two groups of completers are comparable. However, it is worth 
mentioning that the partial completers seemed to have a higher level of symptoms than the full completers at baseline, which may impede their progress. Future studies could consider adjusting the guidance model to increase the participants' engagement in the digital program, which might lead to a better treatment outcome (47).

Attrition is typically greater in non-psychiatric samples and in interventions without real-person feedback and mood monitoring, compared with their counterparts (46). Although this is a common issue in digital mental health interventions, it can severely hinder the scalability and adoption of these interventions $(1,46)$. Two features of SbS, minimal guidance and mood monitoring, might play an important role to motivate the user to continue, assist users to overcome difficulties in completion of the intervention, and offer feedback and overview of user's mood over time $(17,48)$. In addition, the guidance model is critical in digital interventions for people with a higher level of depression as their greater improvement was associated with guided intervention while people with a subthreshold level of depression had similar gains with or without guidance during their intervention (47). These features were also universally mentioned and greatly accepted by the participants in the qualitative interview. Consistent with other adapted versions of the Step-by-Step program, the Chinese version showed preliminary evidence of significant improvement in depressive and anxiety symptoms, and client-defined stress $(20,41)$. Future studies that specifically target users who are seeking mental health support may yield greater completion rates.

Qualitative data provided insights into how the program can be improved. Participants suggested that study recruitment was sufficient as we adopted a variety of strategies to promote it within the residential college, including posters, mass email invitations, referrals, in-class recruitment, posts in social networking applications, and a booth in residential college events. It is recognized that self-stigma might hinder students from using the program (7). Based on previous work (49), recruitment strategies incorporated into this study attempted to use a stigma-free approach in promotion and recruitment to make the intervention or program more friendly and acceptable. Our study framed the program as a tool to help improve mood and academic stress, and most of the recruitment events were done in class, which was considered as stigma-free based on feedback from a participant because most of the students were doing the same task while another participant who participated in feedback session also reminded the recruitment team that outreach and recruitment should target students who need mental health support specifically.

Similar to Heim et al. (20), Chinese University students recommended more engaging activities and features in the app. Suggestions and recommendations from users were carefully considered before program refinement and the conduct of a definitive RCT (24). Changes made for the RCT included providing a download link for users who did not have an application download store on their device, adjusting the time used in the program for the time zone where the program was delivered and not too late, changing the topic selection in the chatroom to show up for the first message a user sent and would be deactivated for subsequent messages within a certain timeframe, offering notification for session unlock events, enabling control condition and randomization, providing a Chinese version of email reminders, increasing the number of seats in the app for RCT to allow more people to be screened, and changing the PHQ-9 cutoff used in screening to a score of 5 to reflect the range of distress in the population and to allow more participants to participate. Detailed qualitative information based on RE-AIM should be reported in the definitive RCT to thoroughly evaluate the entire program implementation process.

The current study has several limitations. Although a feasibility or pilot study is common practice and recommended for evaluation of recruitment potential, preliminary effect size and sample size estimations, and safety assessment before running the main trial, the results should be cautiously interpreted (50), and the effect sizes observed among completers may not be observed in the full trial. The current study design was an uncontrolled, single-arm pilot trial with a small sample size and not fully powered to detect significant changes over time. Also, the uncontrolled feasibility study was unable to inform the subsequent RCT regarding dropout and their reasons in the active control group, which is brief psychoeducation with a contact list of community services. Hence, future studies need to take a more conservative approach to estimate the actual sample size needed in the definitive RCT and take proper measures to reduce dropout in the control group. In addition, the current study piloted SbS in one residential college in one University, which limits generalizability. However, it fulfilled the purpose of the current study-to test the intervention and study procedure in order to inform the conduct of a definitive RCT. Our results revealed that a larger proportion of the participants were female, which might limit the interpretation of the results of this population. Indeed, Hall et al. (32) showed that females reported more mood symptoms than their male counterparts, and this, coupled with a higher proportion of women enrolled in University, can be plausible reasons more female students were included.

Despite the limitations, our study successfully piloted the intervention and the quantitative and qualitative data demonstrated the potential of the program to improve mental health among Chinese University students and provided information for the program amendment, while also supporting that a multi-site collaboration within the academic institution or a larger care setting was feasible. Establishing a strong partnership and collaboration with community stakeholders was beneficial to study implementation and future program sustainability.

\section{DATA AVAILABILITY STATEMENT}

The raw data supporting the conclusions of this article will be made available by the authors, without undue reservation.

\section{ETHICS STATEMENT}

The studies involving human participants were reviewed and approved by University of Macau Research Ethics Board. The 
patients/participants provided their written informed consent to participate in this study.

\section{AUTHOR CONTRIBUTIONS}

$\mathrm{BH}$ conceptualized the project. $\mathrm{BH}$ and $\mathrm{AL}$ supervised the study. $\mathrm{HS}, \mathrm{AL}$, and $\mathrm{BH}$ conducted the study with the support of $\mathrm{IH}, \mathrm{SB}$, $\mathrm{ES}$, and WC. HS organized the database, conducted qualitative interviews, and performed data analysis. $\mathrm{BH}$ and WC supervised data analysis. HS wrote the first draft of the manuscript. IH, SB, $\mathrm{ES}, \mathrm{MW}, \mathrm{WC}, \mathrm{AL}$, and $\mathrm{BH}$ carefully reviewed and edited the manuscript. All authors contributed to manuscript revision, read, and approved the submitted version.

\section{FUNDING}

This work was funded by Macau Foundation and Macao SAR Government and RSTKO (PI: Hall) MYRG2018-00241-FSS.

\section{ACKNOWLEDGMENTS}

We would like to thank Mark van Ommeren and Ken Carswell from the World Health Organization, and Martin Vandendyk, from the WHO Western Pacific Regional Office for their support

\section{REFERENCES}

1. Lattie EG, Adkins EC, Winquist N, Stiles-Shields C, Wafford QE, Graham AK. Digital mental health interventions for depression, anxiety, and enhancement of psychological well-being among college students: systematic review. J Med Internet Res. (2019) 21:e12869. doi: 10.2196/12869

2. Harrer M, Adam SH, Fleischmann RJ, Baumeister H, Auerbach R, Bruffaerts $\mathrm{R}$, et al. Effectiveness of an internet- and app-based intervention for college students with elevated stress: randomized controlled trial. J Med Internet Res. (2018) 20:e136. doi: 10.2196/jmir.9293

3. Cuijpers P, Kleiboer A, Karyotaki E, Riper H. Internet and mobile interventions for depression: opportunities and challenges. Depress Anxiety. (2017) 34:596-602. doi: 10.1002/da.22641

4. Linardon J, Cuijpers $\mathrm{P}$, Carlbring $\mathrm{P}$, Messer M, Fuller-Tyszkiewicz M. The efficacy of app-supported smartphone interventions for mental health problems: a meta-analysis of randomized controlled trials. World Psychiatry. (2019) 18:325-36. doi: 10.1002/wps.20673

5. Rudd BN, Beidas RS. Digital mental health: the answer to the global mental health crisis? JMIR Ment Health. (2020) 7:e18472. doi: 10.2196/18472

6. Clement S, Schauman O, Graham T, Maggioni F, Evans-Lacko S, Bezborodovs $\mathrm{N}$, et al. What is the impact of mental health-related stigma on help-seeking? A systematic review of quantitative and qualitative studies. Psychol Med. (2015) 45:11-27. doi: 10.1017/S0033291714000129

7. Shi W, Hall BJ. Help-seeking intention among Chinese college students exposed to a natural disaster: an application of an extended theory of planned behavior (E-TPB). Soc Psychiatry Psychiatr Epidemiol. (2021) 56:1273-82. doi: 10.1007/s00127-020-01993-8

8. Fu Z, Burger H, Arjadi R, Bockting CLH. Effectiveness of digital psychological interventions for mental health problems in low-income and middle-income countries: a systematic review and meta-analysis. Lancet Psychiatry. (2020) 7:851-64. doi: 10.1016/S2215-0366(20)30256-X

9. Zhang $X$, Lewis S, Firth J, Chen X, Bucci S. Digital mental health in China: a systematic review. Psychol Med. (2021) 51:1-19. doi: 10.1017/S0033291721003731

10. Wood D, Crapnell T, Lau L, Bennett A, Lotstein D, Ferris M, et al. Emerging Adulthood as a Critical Stage in the Life Course. In: Halfon N, Forrest in adapting and trialing Step-by-Step in China. We would also like to thank Rainbow Leong and Marion $\mathrm{Chu}$ from the Student Counseling Section of Student Affairs Office of University of Macau for providing clinical training. We also would like to thank Kit Thompson from Moon Chun Memorial College-University of Macau, for allowing us to recruit peer counselors as e-helpers and study participants in the college. We are grateful to our research assistants (Nancy, Evelyn, Penny, Kit, and Helen), and the group of peer counselors (Sheldon, Rainy, Tissier, Ben, Tina, Michelle, Nora, Christine, Joiy, Claris, Esther, and Kiki), and clinical intern (Franky) to help with recruitment and their work as e-helpers. We would like to thank Evelyn for her assistance in content analysis. We would like to thank Jan and his team at Hybrid Heroes for their work on the app development. We would like to thank Henry Lao, Leah Lin, and Josephine Wong from the Macau Society of Registered Psychotherapists who created the audio exercises.

\section{SUPPLEMENTARY MATERIAL}

The Supplementary Material for this article can be found online at: https://www.frontiersin.org/articles/10.3389/fpsyt. 2021.812667/full\#supplementary-material

CB, Lerner RM, Faustman EM, editors. Handbook of Life Course Health Development. Cham: Springer International Publishing; 2018. p. 123-43. doi: 10.1007/978-3-319-47143-3_7

11. Beiter R, Nash R, McCrady M, Rhoades D, Linscomb M, Clarahan $\mathrm{M}$, et al. The prevalence and correlates of depression, anxiety, and stress in a sample of college students. J Affect Disord. (2015) 173:90-6. doi: 10.1016/j.jad.2014.10.054

12. Gao W, Ping S, Liu X. Gender differences in depression, anxiety, and stress among college students: a longitudinal study from China. J Affect Disord. (2020) 263:292-300. doi: 10.1016/j.jad.2019.11.121

13. Ramón-Arbués E, Gea-Caballero V, Granada-López JM, Juárez-Vela R, Pellicer-García B, Antón-Solanas I. The prevalence of depression, anxiety and stress and their associated factors in college students. Int J Environ Res Public Health. (2020) 17:7001. doi: 10.3390/ijerph17197001

14. Gao L, Xie Y, Jia C, Wang W. Prevalence of depression among Chinese University students: a systematic review and meta-analysis. Sci Rep. (2020) 10:15897. doi: 10.1038/s41598-020-72998-1

15. Lei XY, Xiao LM, Liu YN, Li YM. Prevalence of depression among Chinese University Students: a meta-analysis. PLoS ONE. (2016) 11:e0153454. doi: 10.1371/journal.pone.0153454

16. Chi XL, Becker B, Yu Q, Willeit P, Jiao C, Huang LY, et al. Prevalence and psychosocial correlates of mental health outcomes among Chinese College Students during the Coronavirus Disease (COVID-19) pandemic. Front Psychiatry. (2020) 11:803. doi: 10.3389/fpsyt.2020.00803

17. Carswell K, Harper-Shehadeh M, Watts S, Van't Hof E, Abi Ramia J, Heim E, et al. Step-by-Step: a new WHO digital mental health intervention for depression. Mhealth. (2018) 4:34. doi: 10.21037/mhealth.2018.08.01

18. Abi Ramia J, Harper Shehadeh M, Kheir W, Zoghbi E, Watts S, Heim E, et al. Community cognitive interviewing to inform local adaptations of an e-mental health intervention in Lebanon. Glob Ment Health. (2018) 5:e39. doi: $10.1017 /$ gmh.2018.29

19. Burchert S, Alkneme MS, Bird M, Carswell K, Cuijpers P, Hansen P, et al. Usercentered app adaptation of a low-intensity e-mental health intervention for syrian refugees. Front Psychiatry. (2019) 9:663. doi: 10.3389/fpsyt.2018.00663

20. Heim E, Ramia JA, Hana RA, Burchert S, Carswell K, Cornelisz I, et al. Stepby-step: feasibility randomised controlled trial of a mobile-based intervention 
for depression among populations affected by adversity in Lebanon. Internet Intervent. (2021) 24:100380. doi: 10.1016/j.invent.2021.100380

21. Garabiles MR, Harper Shehadeh M, Hall BJ. Cultural adaptation of a scalable world health organization e-mental health program for overseas Filipino workers. JMIR Form Res. (2019) 3:e11600. doi: 10.2196/11600

22. Shala M, Morina N, Burchert S, Cerga-Pashoja A, Knaevelsrud C, Maercker A, et al. Cultural adaptation of Hap-pas-Hapi, an internet and mobile-based intervention for the treatment of psychological distress among Albanian migrants in Switzerland and Germany. Internet Intervent. (2020) 21:100339. doi: 10.1016/j.invent.2020.100339

23. Sit HF, Ling R, Lam AIF, Chen W, Latkin CA, Hall BJ. The cultural adaptation of step-by-step: an intervention to address depression among Chinese young adults. Front Psychiatry. (2020) 11:650. doi: 10.3389/fpsyt.2020. 00650

24. Sit HF, Li G, Chen W, Sou EKL, Wong M, Buchert S, et al. A protocol for a type 1 effectiveness implementation randomized controlled trial of the WHO digital mental health intervention Step-by-Step to address depression among Chinese young adults in Macao (SAR) China (Under Review).

25. Medical Research Council. Developing and Evaluating Complex Interventions: Following Considerable Development in the Field Since 2006, MRC and NIHR Have Jointly Commissioned an Update of This Guidance to be Published in 2019. London: Medical Research Council (2019).

26. Glasgow RE, Harden SM, Gaglio B, Rabin B, Smith M, Porter GC, et al. RE-AIM planning and evaluation framework: adapting to new science and practice with a 20-year review. Front Public Health. (2019) 7:64. doi: 10.3389/fpubh.2019.00064

27. Glasgow RE, Vogt TM, Boles SM. Evaluating the public health impact of health promotion interventions: the RE-AIM framework. Am J Public Health. (1999) 89:1322-7. doi: 10.2105/AJPH.89.9.1322

28. Eldridge SM, Chan CL, Campbell MJ, Bond CM, Hopewell S, Thabane L, et al. CONSORT 2010 statement: extension to randomised pilot and feasibility trials. BMJ. (2016) 355:i5239. doi: 10.1136/bmj.i5239

29. Dua T, Barbui C, Clark N, Fleischmann A, Poznyak V, van Ommeren $\mathrm{M}$, et al. Evidence-based guidelines for mental, neurological, and substance use disorders in low- and middle-income countries: summary of WHO recommendations. PLoS Med. (2011) 8:e1001122. doi: 10.1371/journal.pmed.1001122

30. Pearson N, Naylor P-J, Ashe MC, Fernandez M, Yoong SL, Wolfenden L. Guidance for conducting feasibility and pilot studies for implementation trials. Pilot Feasibility Stud. (2020) 6:167. doi: 10.1186/s40814-02000634-w

31. Kroenke K, Spitzer RL, Williams JB. The PHQ-9: validity of a brief depression severity measure. J Gen Intern Med. (2001) 16:606-13. doi: 10.1046/j.1525-1497.2001.016009606.x

32. Hall BJ, Lam AIF, Wu TL, Hou WK, Latkin C, Galea S. The epidemiology of current depression in Macau, China: towards a plan for mental health action. Soc Psychiatry Psychiatr Epidemiol. (2017) 52:1227-35. doi: $10.1007 / \mathrm{s} 00127-017-1415-8$

33. Lau KM, Hou WK, Hall BJ, Canetti D, Ng SM, Lam AIF, et al. Social media and mental health in democracy movement in Hong Kong: a population-based study. Comput Hum Behav. (2016) 64:656-62. doi: 10.1016/j.chb.2016.07.028

34. Wang W, Bian Q, Zhao Y, Li X, Wang W, Du J, et al. Reliability and validity of the Chinese version of the Patient Health Questionnaire (PHQ-9) in the general population. Gen Hosp Psychiatry. (2014) 36:539-44. doi: 10.1016/j.genhosppsych.2014.05.021

35. Spitzer RL, Kroenke K, Williams JB, Lowe B. A brief measure for assessing generalized anxiety disorder: the GAD-7. Arch Intern Med. (2006) 166:1092-7. doi: 10.1001/archinte.166.10.1092

36. He X, Li C, Qian J, Cui H, Wu W. Reliability and validity of a generalized anxiety disorder scale in general hospital outpatients. Shanghai Arch Psychiatry. (2010) 22:200-3. doi: 10.3969/j.issn.1002-0829.2010.04.002

37. Tong X, An D, McGonigal A, Park SP, Zhou D. Validation of the generalized anxiety disorder-7 (GAD-7) among Chinese people with epilepsy. Epilepsy Res. (2016) 120:31-6. doi: 10.1016/j.eplepsyres.2015.11.019
38. Volinn E, Yang B, He J, Sheng X, Ying J, Volinn W, et al. West China hospital set of measures in Chinese to evaluate back pain treatment. Pain Med. (2010) 11:637-47. doi: 10.1111/j.1526-4637.2010.00846.x

39. Bech P, Olsen LR, Kjoller M, Rasmussen NK. Measuring well-being rather than the absence of distress symptoms: a comparison of the SF-36 mental health subscale and the WHO-Five well-being scale. Int J Meth Psych Res. (2003) 12:85-91. doi: 10.1002/mpr.145

40. Ashworth M, Shepherd M, Christey J, Matthews V, Wright K, Parmentier $\mathrm{H}$, et al. A client-generated psychometric instrument: the development of "PSYCHLOPS". Counsel Psychother Res. (2004) 4:27-31. doi: 10.1080/14733140412331383913

41. Harper Shehadeh MJ, Abi Ramia J, Cuijpers P, El Chammay R, Heim E, Kheir W, et al. Step-by-step, an e-mental health intervention for depression: a mixed methods pilot study from Lebanon. Front Psychiatry. (2020) 10:986. doi: $10.3389 /$ fpsyt.2019.00986

42. StataCorp. Stata Statistical Software: Release 16. College Station, TX: StataCorp LLC (2019).

43. Duggan C, Parry G, McMurran M, Davidson K, Dennis J. The recording of adverse events from psychological treatments in clinical trials: evidence from a review of NIHR-funded trials. Trials. (2014) 15:335. doi: 10.1186/1745-6215-15-335

44. Melville KM, Casey LM, Kavanagh DJ. Dropout from Internet-based treatment for psychological disorders. Br J Clin Psychol. (2010) 49:455-71. doi: $10.1348 / 014466509 X 472138$

45. Meyerowitz-Katz G, Ravi S, Arnolda L, Feng X, Maberly G, Astell-Burt T. Rates of attrition and dropout in app-based interventions for chronic disease: systematic review and meta-analysis. J Med Internet Res. (2020) 22:e20283. doi: $10.2196 / 20283$

46. Torous J, Lipschitz J, Ng M, Firth J. Dropout rates in clinical trials of smartphone apps for depressive symptoms: a systematic review and metaanalysis. J Affect Disord. (2020) 263:413-9. doi: 10.1016/j.jad.2019.11.167

47. Karyotaki E, Efthimiou O, Miguel C, Bermpohl FMG, Furukawa TA, Cuijpers $\mathrm{P}$, et al. Internet-based cognitive behavioral therapy for depression: a systematic review and individual patient data network meta-analysis. JAMA Psychiatry. (2021) 78:361-71. doi: 10.1001/jamapsychiatry.2020.4364

48. Andersson G, Titov N. Advantages and limitations of Internet-based interventions for common mental disorders. World Psychiatry. (2014) 13:4-11. doi: 10.1002/wps.20083

49. Ling R, Sit HF, Balaji S, Lam AIF, Latkin CA, Tucker JD, et al. Crowdsourcing to design a marketing package to promote a WHO digital mental health intervention among Chinese young adults. Internet Intervent. (2021) 25:100397. doi: 10.1016/j.invent.2021.100397

50. Thabane L, Ma J, Chu R, Cheng J, Ismaila A, Rios LP, et al. A tutorial on pilot studies: the what, why and how. BMC Med Res Methodol. (2010) 10:1. doi: $10.1186 / 1471-2288-10-1$

Conflict of Interest: The authors declare that the research was conducted in the absence of any commercial or financial relationships that could be construed as a potential conflict of interest.

Publisher's Note: All claims expressed in this article are solely those of the authors and do not necessarily represent those of their affiliated organizations, or those of the publisher, the editors and the reviewers. Any product that may be evaluated in this article, or claim that may be made by its manufacturer, is not guaranteed or endorsed by the publisher.

Copyright $\odot 2022$ Sit, Hong, Burchert, Sou, Wong, Chen, Lam and Hall. This is an open-access article distributed under the terms of the Creative Commons Attribution License (CC BY). The use, distribution or reproduction in other forums is permitted, provided the original author(s) and the copyright owner $(s)$ are credited and that the original publication in this journal is cited, in accordance with accepted academic practice. No use, distribution or reproduction is permitted which does not comply with these terms. 